\title{
Metabolism of conjugated sterols in eggplant. Part 1. UDP-glucose : sterol glucosyltransferase
}

\author{
Anna Potocka and Jan Zimowski ${ }^{\bowtie}$ \\ Institute of Biochemistry, Warsaw University, Warszawa, Poland
}

Received: 16 January, 2007; revised: 09 November, 2007; accepted: 13 December, 2007

available on-line: 16 January, 2008

\begin{abstract}
A membrane-bound UDP-glucose : sterol glucosyltransferase from Solanum melongena (eggplant) leaves was partially purified and its specificity as well as molecular and kinetic properties were defined. Among a wide spectrum of 3-OH steroids (i.e. typical plant sterols, androstane, pregnane and cholestane derivatives, steroidal alkaloids and sapogenins) and triterpenic alcohols, the highest activity was found with 22-oxycholesterol. UDP-glucose appeared to be the best sugar donor. The enzyme preparation was also able to utilize UDP-galactose, TDP-glucose and CDP-glucose as a sugar source for sterol glucosylation, however, at distinctly lower rates. The investigated glucosyltrasferase was stimulated by 2-mercaptoethanol, Triton X-100 and negatively charged phospholipids, and inhibited in the presence of UDP, mono-, di- and triacylglycerols, divalent cations such as $\mathrm{Zn}^{2+}, \mathrm{Co}^{2+}$, high ionic strength, cholesteryl glucoside, galactoside and xyloside and some amino acid-modifying reagents (SITS, DIDS, PLP, DEPC, pCMBS, NEM, WRK and HNB). Our results suggest that unmodified residues of lysine, tryptophan, cysteine, histidine and dicarboxylic amino acids are essential for full enzymatic activity and indicate that a glutamic (or aspartic) acid residue is necessary for the binding of sugar donor, i.e. UDP-glucose in the active site of the GT-ase while histidine and cysteine residues are both important for the binding of the nucleotide-sugar as well as of the steroidal aglycone.
\end{abstract}

Keywords: plant sterols, Solanum melongena, steryl glucoside, UDP-glucose : sterol glucosyltransferase

\section{INTRODUCTION}

Apart from free sterols, their conjugated forms, i.e. steryl esters, steryl 3-O- $\beta$-D-monoglycosides (SG) and steryl 6'-O-acyl- $\beta$-D-glycosides are widely distributed common constituents in higher plant cells (see Scheme). They were also found in ferns, mosses and fungi, while their presence in algae and prokaryotes has been reported in a few cases only (Wojciechowski, 1991; Tannes et al., 2000). Plant SG usually consist of a mixture of individual "molecular species" differing in sterol and/or saccharide moiety. The predominating sugar moiety found

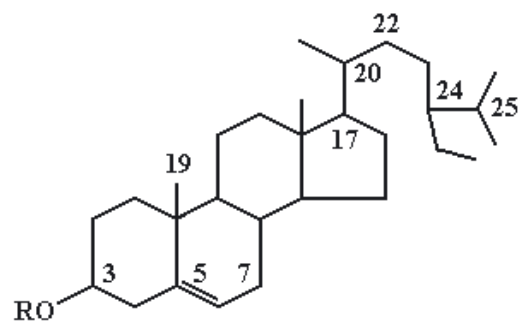

$\mathbf{R}=\mathbf{H}$

$\mathbf{R}=$ acyl residue

$\mathbf{R}=$ gluc opyranose

$\mathbf{R}=6^{\prime}-O$-acylglucopyranose sitosterol sitosteryl ester sitosteryl glucoside sitosteryl 6'- $O$-acylglucopyranose

${ }^{\triangle}$ Corresponding author: Jan Zimowski, Instytut Biochemii UW, I. Miecznikowa 1, 02-096 Warszawa, Poland; phone: (48) 22 554 3404; fax: (48) 22554 3221; e-mail: janjzim@biol.uw.edu.pl

Abbreviations: DEPC, diethylpyrocarbonate; DGDG, digalactosyldiacylglycerols; DIDS, 4,4'-diisothiocyanatostilbene-2,2'disulfonic acid; DTE, dithioerythritol; DTT, dithiothreitol; GTase(s), glucosyltransferase(s); HNB, 2-hydroxy-5-nitrobenzyl bromide (Koshland I Reagent); MGDG, monogalactosyldiacylglycerols; NAI, N-acetylimidazole; NEM, N-ethylmaleimide; PA, phosphatidic acid; PC, phosphatidylcholine; pCMBS, p-chloromercuribenzenesulfonic acid; PE, phosphatidylethanolamine; PG, phenylglyoxal; PI, phosphatidylinositol; PLP, pyridoxal 5'-phosphate; PMSF, phenylmethylsulfonyl fluoride; SG, steryl glucosides; SITS, 4-acetamido-4'-isothiocyanatostilbene-2,2'-disulfonic acid; WRK, Woodward's Reagent K. 
in SG is glucose, but galactose, xylose and others also occur (Jares et al., 1990; Wojciechowski, 1991; Jahan et al., 1995; Casabuono \& Pomilo, 1997). Besides the monoglycoside steryl derivatives, glycosides with an oligosaccharide chain (up to five sugar residues) have been found (Fujino \& Ohnishi, 1979; Kojima et al., 1989). SG and their 6'-O-acyl derivatives in general occur in small amounts, but plants from the Solanum genus, e.g. S. tuberosum, S. lycopersicon, S. dulcamara, demonstrate a unique abundance of SG and ASG (acetylated steryl glycoside), as the glycosylated forms of sterols make up to $30-50 \%$ of total sterols in these plants (Duperon et al., 1984; Duperon \& Duperon, 1989; Zimowski \& Wojciechowski, 1996). In some papers one can find suggestions that SG present in the above plants are a mixture of steryl $\beta$-D-monoglucosides and $\beta$-D-monogalactosides (Duperon et al., 1984; Duperon \& Duperon, 1989).

Enzymatic glucosylation of sterols in the presence of UDP-glucose has been demonstrated in cell-free preparations and in various crude membraneous fractions obtained from vascular plants (Wojciechowski \& Nguyen Van Uon, 1975; Fang \& Baisted, 1976; Baisted, 1978; Wojciechowski et al., 1979; Pączkowski et al., 1990; Wojciechowski, 1991; Zimowski 1992), green algae (Forsee et al., 1974; Hopp et al., 1978) and non-photosynthesizing microorganisms (Esders \& Light 1972; Wojciechowski et al., 1979; Warnecke et al., 1999). Only in a few cases have the respective enzymes been partially purified and characterized (Wojciechowski \& Nguyen Van Uon, 1975; Wojciechowski et al., 1979; Yoshikawa \& Furuya 1979; Ullmann et al., 1993; Warnecke \& Heinz, 1994). Recently, sterol GTases from oat, Arabidopsis thaliana and Saccharomyces cerevisiae, Candida albicans, Pichia pastoris and Dictyostelium discoideum were cloned and expressed in Escherichia coli (Warnecke et al., 1997, 1999).

Whether SG are active metabolic compounds or not remains an unsolved problem. Some hypotheses consider these conjugates as final products of sterol metabolism or reserve forms of sterols in plant cell. Others describe them as metabolically active substances, which may participate in intracellular transport of glucose (Murakami-Murofushi et al., 1997) or in specific transglucosylation processes, e.g. in cellulose (Peng et al., 2002; Peters, 2002) or cerebroside biosynthesis (Cantatore et al., 2000). On the other hand, the processes of sterol glucosylationdeglucosylation or sterol glucoside acylation-deacylation, which take place in membranes, can play a significant role in the properties of these membranes.

It is expected that UDP-glucose : sterol GTase, as an enzyme involved in the above-mentioned transformations, may play a significant physiological role. Glucosylation of free sterols represents a meta- bolic process most probably ubiquitous in plants and may participate in the regulating of free sterol concentration in the cell. Except the GTase from oat, data about basic molecular and catalytic properties of the enzyme involved in sterol glucosylation is still lacking. In the presented paper we determined biochemical properties of UDP-glucose : sterol GTase isolated from eggplant leaves.

\section{MATERIAL AND METHODS}

Plant material. Eggplant (Solanum melongena L., cv Black Beauty) plants were grown on artificial support (Perlite) in a greenhouse at a $16 / 8 \mathrm{~h}$ photoperiod, at $25 / 20^{\circ} \mathrm{C}$ day/night. Leaves were cut off from 9-week old plants.

Enzyme preparation. Fresh leaves of eggplant (100 g fresh mass) were homogenized with $200 \mathrm{ml}$ of ice-cold $0.1 \mathrm{M}$ Tris/ $\mathrm{HCl}$ buffer, $\mathrm{pH}$ 7.3, containing $10 \mathrm{mM}$ 2-mercaptoethanol (buffer A). The homogenate was filtered through cheesecloth and centrifuged at $3000 \times g(10 \mathrm{~min})$. Supernatant was centrifuged at $20000 \times g(20 \mathrm{~min})$ and the $20000 \times g$ pellet (the membrane fraction) was resuspended in buffer A and added dropwise to a 10-fold volume of cold (minus $20^{\circ} \mathrm{C}$ ) acetone so "acetone powder I" was obtained as previously described (Wojciechowski et al., 1979). "Acetone powder I" was resuspended in buffer A containing $0.1 \%$ Triton X-100 (buffer B) and centrifuged at $20000 \times g(20 \mathrm{~min})$. Supernatant (partly delipidated enzyme preparation) was added dropwise to a 10 -fold volume of acetone $\left(4^{\circ} \mathrm{C}\right)$ and "acetone powder II" was obtained as previously described (Wojciechowski et al., 1979).

Glucosyltransferase purification. Gel filtration was carried out on a Sephadex G-150 column $(2 \times 40 \mathrm{~cm})$ equilibrated with buffer B. The enzyme preparation (acetone powder II, $10 \mathrm{mg} / \mathrm{ml}$ ) was applied onto the column and eluted with buffer B at a flow rate of $0.4 \mathrm{ml} / \mathrm{min}$. Fractions exhibiting the highest GTase activity were combined (12-20 ml) and then applied onto a Q-Sepharose (Sigma) column $(1 \times 3 \mathrm{~cm})$, previously equilibrated with buffer $B$ and eluted at a flow rate of $0.4 \mathrm{ml} / \mathrm{min}$. Unbound proteins were washed out from the column with 30 $\mathrm{ml}$ of buffer $\mathrm{B}$ and then the column was developed

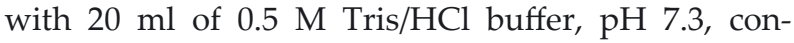
taining $10 \mathrm{mM}$ 2-mercaptoethanol and $0.1 \%$ Triton $\mathrm{X}-100$ (flow rate $0.4 \mathrm{ml} / \mathrm{min}$ ). This fraction was dialyzed overnight against buffer B and GTase activity was assayed.

Glucosyltransferase assay. The standard reaction mixture contained in a total volume of $0.52 \mathrm{ml}$ : enzyme preparation $(50 \mu \mathrm{g}$ protein in $0.5 \mathrm{ml}$ of buffer B); $25 \mathrm{nmol}$ of sitosterol (or another sugar acceptor) or $\left[4-{ }^{14} \mathrm{C}\right]$ cholesterol (Reachim, $3.0 \times 10^{5} \mathrm{dpm}, 2.5$ 
$\mathrm{nmol}$ ) in $0.01 \mathrm{ml}$ ethanol, and UDP- $\left[{ }^{14} \mathrm{C}\right]$ glucose (Amersham, $2.0 \times 10^{5} \mathrm{dpm}, 6 \mathrm{nmol}$ ) or unlabelled UDPglucose (Sigma, or another sugar donor, $15 \mathrm{nmol}$ ) in $0.01 \mathrm{ml} \mathrm{50 \%} \mathrm{ethanol.} \mathrm{Once} \mathrm{UDP-glucose} \mathrm{was} \mathrm{added,}$ the reaction was run at $30^{\circ} \mathrm{C}$ for $30 \mathrm{~min}$ and stopped by adding of $1 \mathrm{ml}$ of methanol and heating for $3 \mathrm{~min}$ in a boiling water bath. Subsequently samples were extracted with $4 \mathrm{ml}$ of 1-butanol saturated with water ( 3 times) and the butanolic extracts were washed several times with water saturated with 1-butanol. The samples were air-dried and applied on silica gel plates (Merck) and developed with chloroform/ methanol $(9: 1, \mathrm{v} / \mathrm{v})$, as a solvent. Labelled products were localized by autoradiography and their chromatographic mobility was compared with those of authentic reference compounds: 3-O- $\beta$-D-monoglucopyranoside of sitosterol $\left(\mathrm{R}_{\mathrm{F}}=0.32\right)$ and 3-O- $\beta$-Dmonogalactopyranoside of sitosterol $\left(R_{F}=0.27\right)$. The radioactivity measurements of butanolic extracts or purified radioactive products were carried out as previously described (Zimowski, 1991).

Other methods. Unlabelled steroidal monoglycosides, i.e. cholesteryl, sitosteryl, stigmasteryl 3$O-\beta$-D-monoglucopyranosides or 3-O- $\beta$-D-monogalactopyranosides were obtained as previously described (Janiszowska et al., 1980).

\section{RESULTS}

Preliminary incubations performed in the presence of $\left[{ }^{14} \mathrm{C}\right]$ cholesterol and non-radioactive UDP-glucose with homogenate obtained from 9week old S. melongena leaves indicated that this preparation was able to catalyze the formation of labelled cholesteryl glucoside. Similar incubations with subcellular fractions separated by differential centrifugation indicated that the investigated GTase activity copurified with membrane fractions. The fraction sedimenting between $3000-20000 \times g$ was the most active, exhibiting a 3-4-times higher total activity than the remaining membrane fractions.

The investigated enzyme was partially purified by gel filtration on Sephadex G-150 and by filtration on Q-Sepharose. The purified enzyme preparation showed a 21 -fold increase of specific activity and a 2-fold decrease of total activity comparing with the crude homogenate.

Molecular and kinetic properties of UDP-glucose : sterol glucosyltransferase from eggplant leaves

The partially purified enzyme exhibits apparent $K_{\mathrm{m}}$ values, $14.29 \mu \mathrm{M}$ for UDP-glucose, $1.43 \mu \mathrm{M}$ for sitosterol. Divalent metal ions, such as $\mathrm{Mg}^{2+}$, $\mathrm{Ca}^{2+}, \mathrm{Mn}^{2+}$ and some metal chelators (EDTA, EGTA) show no effect on the GTase activity. On the other hand, some heavy metal ions had a strong inhibitory effect $\left(\mathrm{I}_{50}\right.$ for $\mathrm{Zn}^{2+}$ and $\mathrm{Co}^{2+}$ was 0.09 and 0.53 $\mathrm{mM}$, respectively). The GTase activity was strongly inhibited in the presence of UTP, UDP $\left(\mathrm{I}_{50}=0.035\right.$, $0.065 \mathrm{mM}$, respectively) and some amino acid-modifying reagents i.e. SITS, DIDS, PLP, DEPC, pCMBS, NEM, WRK and HNB ( $I_{50}$ value was 0.28, 0.021, $1.43,1.0,0.003,0.043,0.8$, and $1.2 \mathrm{mM}$, respectively). The enzyme catalyzing the biosynthesis of SG was also sensitive to higher ionic strength of the incubation medium $\left(\mathrm{I}_{50}\right.$ for $\mathrm{NaF}$ and $\mathrm{NaCl}$ was $0.52 \mathrm{M}$ and $0.29 \mathrm{M}$, respectively). Unlabelled (cold) steryl glycosides, e.g. cholesteryl glucoside, galactoside and xyloside added to the incubation mixtures in the presence of cholesterol and UDP- $\left[{ }^{14} \mathrm{C}\right]$ glucose caused a considerable decrease of the formation of labelled cholesteryl glucoside $\left(\mathrm{I}_{50}=4.6,10.8,61.8 \mu \mathrm{M}\right.$, respectively). Detergents commonly used to solubilize and/ or to activate membrane-bound enzymes, e.g. Tween 20, Tween 40, Tween 60, Tween 80, Tyloxapol, sodium deoxycholate showed practically no effect on the investigated GT-ase. On the other hand, n-octyl thioglucoside irreversibly inhibited $\left(\mathrm{I}_{50}=4.8 \mathrm{mM}\right)$ while Triton X-100 strongly activated (approx. 130fold at $0.1 \%$ conc.) the studied enzyme.

Specificity of UDP-glucose : sterol glucosyltransferase from eggplant leaves towards sugar moiety acceptor and donor

The partially purified enzyme preparation was used to study the substrate specificity of the investigated GTase towards the sugar moiety acceptor in the presence of UDP- $\left[{ }^{14} \mathrm{C}\right]$ glucose. Typical sterols and their oxidized derivatives, some $\mathrm{C}_{19}$ and $\mathrm{C}_{21}$ steroids, steroidal alkaloids, sapogenins and triterpenic alcohols were tested as potential sugar acceptors (Table 1). In all cases when incorporation of radioactivity into the butanol extract was found, TLC analysis with subsequent autoradiography showed the presence of a single labelled product with chromatographic mobility expected for the monoglucoside of a given steroid. Among the natural plant sterols tested sitosterol was the best sugar acceptor. A comparison of the configuration of the $\mathrm{OH}$ - group at C-3 and/or the coupling of A and B rings (cis or trans) in saturated isomers of $\mathrm{C}_{27}$-stanols (cholestanol, epicholestanol, epicoprostanol and coprostanol) showed that only cholestanol served as sugar acceptor. Thiocholesterol and triterpenic alcohols were not active as sugar acceptors. The presence and/or the position of double bonds in the steroid molecule seem to play an important role in glucosylation rate. This effect is distinct when comparing the data for glucosylation of sterols differing in the number and/ or position of double bonds. The glucosylation rates 
Table 1. Specificity of UDP-glucose : sterol GTase from S. melonge$n a$ leaves towards glucosyl moiety acceptors

\begin{tabular}{|c|c|}
\hline Acceptor & $\begin{array}{l}\text { Steryl }\left[{ }^{14} \mathrm{C}\right] \text { glucosi- } \\
\text { de formation }(\%)^{*}\end{array}$ \\
\hline \multicolumn{2}{|l|}{ Steroid alkaloids } \\
\hline \multicolumn{2}{|l|}{ a) Spirosolane type } \\
\hline 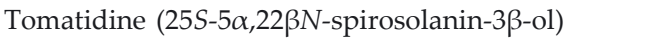 & 2.8 \\
\hline 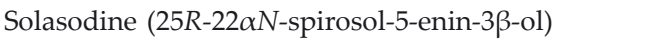 & 0.0 \\
\hline \multicolumn{2}{|l|}{ b) Solanidane type } \\
\hline Solanidine (22S,25S-solanid-5-enin-3 $\beta$-ol) & 0.0 \\
\hline Demissidine $(22 S, 25 S-5 \alpha$-solanidanin-3 $\beta$-ol $)$ & 1.4 \\
\hline \multicolumn{2}{|l|}{ Steroid sapogenin } \\
\hline Nuatigenin (22S,25S-epoxy-furost-5-en-3 $\beta, 26$-diol) & 16.1 \\
\hline Isonuatigenin (25R-spirost-5-en-3 $\beta, 25 \beta$-diol) & 13.4 \\
\hline Hecogenin ( $25 R$ - $5 \alpha$-spirostan-3 $\beta$-ol-12-on) & 6.3 \\
\hline Diosgenin (25R-spirost-5-en-3 $\beta$-ol) & 3.5 \\
\hline 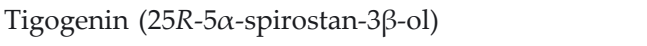 & 2.1 \\
\hline Sarsasapogenin (25S-5 $\beta$-spirostan- $3 \beta$-ol) & 0.0 \\
\hline 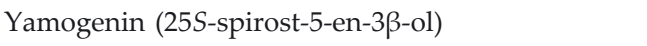 & 0.0 \\
\hline \multicolumn{2}{|l|}{ Sterols and their derivatives } \\
\hline Sitosterol (stigmast-5-en-3 $\beta$-ol) & 100.0 \\
\hline Stigmasterol (stigmasta-5,22-dien-3 $\beta$-ol) & 49.2 \\
\hline Stigmasta-5,24(28)-dien-3 $\beta$-ol & 82.4 \\
\hline Stigmastan-3 $\beta$-ol & 38.7 \\
\hline Cholesterol (cholest-5-en-3 $\beta$-ol) & 98.5 \\
\hline 25-Hydroxycholesterol (cholest-5-en-3 $\beta, 25$-diol) & 89.4 \\
\hline Cholest-5-en-3 $\beta, 20 \alpha$-diol & 90.8 \\
\hline Cholest-5-en-3 $\beta, 19$-diol & 9.2 \\
\hline Cholest-5-en-3 $\beta$-ol-7-on & 5.6 \\
\hline 22-Oxycholesterol (cholest-5-en-22-on-3 $\beta$-ol) & 133.1 \\
\hline $5 \alpha$-Cholest-7-en-3 $\beta$-ol & 14.1 \\
\hline Thiocholesterol (cholest-5-en-3 $\beta$-tiol) & 0.0 \\
\hline $5 \alpha$-Cholestan-3 $\beta$-ol (cholestanol) & 12.0 \\
\hline $5 \beta$-Cholestan-3 $\beta$-ol (coprostanol) & 0.0 \\
\hline $5 \alpha$-Cholestan-3 $\alpha$-ol (epicholestanol) & 0.0 \\
\hline $5 \beta$-Cholestan-3 $\alpha$-ol (epicoprostanol) & 0.0 \\
\hline \multicolumn{2}{|l|}{ Androstane or pregnane derivatives } \\
\hline Androstenolon (androst-5-en-3 $\beta$-ol-17-on) & 16.0 \\
\hline Pregnenolon (pregn-5-en-3 $\beta$-ol-20-on) & 33.1 \\
\hline \multicolumn{2}{|l|}{ Triterpenic alcohols } \\
\hline Lanosterol $\left(4,4^{\prime}, 14\right.$-trimethyl- $5 \alpha$-cholest-8-en-3 $\beta$-ol $)$ & 0.0 \\
\hline$\beta$-Amyrin (olean-12-en-3 $\beta$-ol) & 0.0 \\
\hline
\end{tabular}

*The glucosylation rate for sitosteryl glucosylation (355 fkat) was taken as $100 \%$. Incubations were carried out in the presence of unlabelled sugar moiety acceptor $(25 \mathrm{nmol})$ and UDP- $\left[{ }^{14} \mathrm{C}\right]$ glucose as described in Material and Methods.

of these sterols show the following sequence: $\mathrm{C}_{29} \Delta^{5}$ $>\mathrm{C}_{29} \Delta^{5,24(28)}>\mathrm{C}_{29} \Delta^{5,22}>\mathrm{C}_{29} \Delta^{0}$ and $\mathrm{C}_{27} \Delta^{5}>\mathrm{C}_{27} \Delta^{7}$ $\geq \mathrm{C}_{27} \Delta^{0}$. The modification of sterol side chain structure also has an important effect on glucosylation rate. $\mathrm{C}_{29}$-sterols (i.e. sterols with an additional alkyl group in the side chain at C-24) were better glucose acceptors than $\mathrm{C}_{27}$-sterols (sitosterol versus cholesterol, stigmastanol versus cholestanol). Pregnane and androstane derivatives $\left(\mathrm{C}_{21}\right.$ and $\mathrm{C}_{19}$ steroids, respec- tively) as well as some sapogenins and steroidal alkaloids $\left(\mathrm{C}_{27}\right.$ sterol derivatives) tested were glucosylated at a much lower rate (nuatigenin, isonuatigenin, hecogenin, diosgenin, androstenolon, pregnenolon) or were not glucosylated (sarsasapogenin, yamogenin, solasodine, solanidine). The incorporation of oxygen atom into the sterol molecule side chain had practically no effect (25-hydroxycholesterol, cholest-5-en$3 \beta, 20 \alpha$-diol) or stimulated the appropriate glucoside biosynthesis (22-oxycholesterol). On the other hand, incorporation of oxygen atom into the sterol planar ring system strongly inhibited the formation of the appropriate glucoside (cholest-5-en$3 \beta$-ol-7-on, cholest-5-en-3 $\beta, 19$-diol).

To determine the substrate specificity of the enzyme with respect to the sugar donor, the formation of $\left[{ }^{14} \mathrm{C}\right]$ cholesteryl monoglycosides in the presence of $\left[{ }^{14} \mathrm{C}\right]$ cholesterol and various unlabelled nucleotide-sugars (e.g. UDP-glucose, UDPgalactose, UDP-mannose, UDP-xylose, CDP-glucose, TDP-glucose, ADP-glucose and GDP-glucose) was tested. Our results indicate that only UDP-glucose, UDP-galactose, TDP-glucose and CDP-glucose were sugar donors for glycosylation of $\left[{ }^{14} \mathrm{C}\right]$ cholesterol, however at different rates (48.3, 12.8, 13.0 and 7.5 fkat, respectively). An autoradiographic analysis of labelled product(s) formed in the presence of the above-mentioned nucleotide-sugars showed that in all cases only one radioactive compound with chromatographic mobility of cholesteryl monoglucoside was synthesized.

\section{Effect of chemical compounds selectively modifying amino-acid residues on steryl glucoside formation}

Significant inhibition was obtained in the presence of reagents modifying lysine $\varepsilon-\mathrm{NH}_{2}$ group (DIDS, SITS and PLP), histidine (DEPC), dicarboxylic amino acids (WRK), tryptophan (HNB) and free thiol group in cysteine (pCMBS, NEM) (see above). On the other hand, reagents acting on serine, arginine and tyrosine, i.e. PMSF, PG and NAI, respectively, had no effect on steryl glucoside formation. In order to identify the amino acids involved in substrate binding, the effect of various amino acidmodifying reagents on steryl glucoside biosynthesis was investigated after preincubation of the enzyme preparation with the substrate, i.e. UDP-glucose or 
Table 2. Eggplant GT-ase activity protection in the presence of amino acid-modifying reagents by UDP-glucose or sitosterol.

Enzyme preparation was preincubated for $5 \mathrm{~min}$ with or without sitosterol $(46 \mu \mathrm{M})$ or with or without UDP-[ $\left.{ }^{14} \mathrm{C}\right] \mathrm{glucose}$ (33.5 kBq, final conc. $0.29 \mu \mathrm{M})$. Then modifying reagent was added and preincubation was continued for 10 min. The enzymatic reaction for samples preincubated with sitosterol or UDP-[ $\left[{ }^{14} \mathrm{C}\right]$ glucose was initiated by an addition of labelled UDP-glucose or sitosterol, respectively. For samples preincubated without substrate the reaction was started by the use of equivalent amount of unlabelled sitosterol and UDP-[ $\left.{ }^{14} \mathrm{C}\right]$ glucose.

\begin{tabular}{|c|c|c|c|c|c|}
\hline \multirow{3}{*}{ Amino acid modified } & \multirow{3}{*}{$\begin{array}{l}\text { Amino acid-modify- } \\
\text { ing reagent }\end{array}$} & \multirow{3}{*}{$\begin{array}{l}\text { Concentration of } \\
\text { reagent }(\mathrm{mM})\end{array}$} & \multicolumn{3}{|c|}{ Relative activity of GTase from eggplant leaves $(\%)^{*}$} \\
\hline & & & \multicolumn{3}{|c|}{ Preincubation } \\
\hline & & & $\begin{array}{l}\text { without } \\
\text { substrates }\end{array}$ & $\begin{array}{l}\text { with } \\
\text { sitosterol }\end{array}$ & $\begin{array}{c}\text { with } \\
\text { UDP-glucose }\end{array}$ \\
\hline \multirow{3}{*}{ Lysine } & DIDS & 0.05 & $20 \pm 7$ & $20 \pm 7$ & $27 \pm 4$ \\
\hline & SITS & 0.5 & $17 \pm 7$ & $18 \pm 8$ & $24 \pm 1$ \\
\hline & PLP & 2.0 & $19 \pm 4$ & $24 \pm 3$ & $32 \pm 3$ \\
\hline Cysteine & NEM & 0.1 & $19 \pm 5$ & $37 \pm 1$ & $42 \pm 5$ \\
\hline Histidine & DEPC & 2.0 & $29 \pm 6$ & $57 \pm 7$ & $54 \pm 7$ \\
\hline Tryptophan & HNB & 2.3 & $24 \pm 8$ & $23 \pm 5$ & $24 \pm 9$ \\
\hline Aspartic and glutamic acid & WRK & 2.0 & $26 \pm 7$ & $20 \pm 6$ & $94 \pm 1$ \\
\hline
\end{tabular}

*Enzyme activity without amino acid-modifying reagent was taken as $100 \%$.

sitosterol (Table 2). Noticeable protection of the enzyme against inactivation with NEM or DEPC was found when GTase was preincubated with sitosterol or with UDP-glucose. Preincubation of the enzyme with UDP-glucose, but not with sitosterol, practically completely eliminated the inhibitory effect of WRK.

The influence of some lipids on sterol glucosylation

Preliminary investigations showed that various lipids (phospholipids, glycolipids and acylglycerols) had no effect on sitosteryl glucoside biosynthesis catalyzed by native enzyme preparation (i.e. homogenate or subcellular fractions). In contrast, sitosterol glucosylation was distinctly modified in the presence of lipids when a completely delipidated enzyme preparation was used. Among the lipid tested, an inhibitory effect on the formation of steryl glucoside was observed with acylglycerols, e.g. 1-monopalmitoyl-, 1,2-dipalmitoyl-, 1,3-dipalmitoyl- and tripalmitoylglycerol (27-35\% inhibition), whereas typical chloroplast glycolipids (MGDG and DGDG from wheat) and zwitterionic phospholipids (e.g. synthetic 1,2-dipalmitoyl-PC and 1,2-dipalmitoyl PE) had no and a slightly stimulatory effect, respectively. However, negatively charged phospholipids, i.e. phosphatidylglycerol (synthetic 1,2-dipalmitoyl-), PA (synthetic 1,2-dipalmitoyl-) and PI (from soya bean) significantly stimulated steryl glucoside biosynthesis in comparison with the control without lipid added (175, 146 and 123\% stimulation, respectively). In order to establish whether the above-described effects depended on acyl composition of the phospholipid molecule, several "molecular species" of phosphatidic acids and phosphatidylglycerols differing in fatty acids' chain length or saturation were tested. The obtained results indicated that the chain length of fatty acid residue (from $C_{12}$ to $C_{16}$ in the case of synthetic PA) had no effect on steryl glucoside formation by GT-ase from eggplant leaves. The presence of an unsaturated fatty acid residue in the phosphatidylglycerol molecule evidently stimulated (about 1.6-fold) sterol glucosylation (in the presence of 1,2- $\mathrm{diC}_{18: 0}-$ or $\mathrm{diC}_{18: 1}$ phosphatidylglycerol the rate of formation of sitosteryl glucoside was 522.8 or 849.4 fkat, respectively).

\section{DISCUSSION}

Glucosylation of sterols in the presence of UDP-glucose was demonstrated for the first time 40 years ago with the use of cell-free preparations from immature soybean seeds (Hou et al., 1967). Our results indicate that an enzyme preparation obtained from $S$. melongena leaves by differentil centrifugation is also able to catalyze the formation of steryl glucosides in the presence of exogenously added sugar donor and sugar acceptor (i.e. UDP-glucose and $\left[{ }^{14} \mathrm{C}\right]$ cholesterol or UDP- $\left[{ }^{14} \mathrm{C}\right]$ glucose and sitosterol). The investigated membraneous enzyme, i.e. UDP-glucose : sterol GTase from eggplant, shares some properties with other GTases acting on sterols. All of them are bound with membranes and, except for the enzyme from Physarum polycephalum (Wojciechowski 
\& Nguyen Van Uon, 1975), are strongly activated by the non-ionic detergent Triton X-100 (Wojciechowski, 1991). Solubilization of membrane-bound UDP-glucose : sterol GTase with Triton X-100 (Wojciechowski \& Nguyen Van Uon, 1975; Hopp et al., 1978; Wojciechowski, 1991) or n-octyl- $\beta$-D-thioglucoside (Warnecke \& Heinz, 1994) followed by typical purification methods results in the 30-100-fold or 12500 -fold increase of enzyme activity, respectively. The eggplant GTase was solubilized and strongly activated by Triton X-100 only. The purification procedure with the use of this detergent leads to a 21 -fold increase of the enzyme activity. The n-Octyl derivative cannot be use for eggplant GTase purification as it causes irreversible inhibition of the enzyme activity.

In contrast to a partially purified GTase from pea seedlings (Fang \& Baisted, 1976), the partially purified GTase from eggplant (like the enzyme preparations from Calendula officinalis (Wojciechowski \& Nguyen Van Uon, 1975), potato plant (Zimowski, 1992) or Sinapis alba seedlings (Wojciechowski et al., 1979)) failed to be stimulated by $\mathrm{Mg}^{2+}$ or $\mathrm{Ca}^{2+}$ or by chelating factors. It was shown that most GTases are strongly inhibited by one of the reaction products, i.e. UDP (Wojciechowski, 1991). The GTase from eggplant, apart from UDP, is also inhibited by the second reaction product, i.e. steryl glucoside. The apparent molecular masses of UDP-glucose : sterol GTases range from $56 \mathrm{kDa}$ for oat GTase (Warnecke \& Heinz, 1994) to $140 \mathrm{kDa}$ for the enzyme from white mustard seedlings (Wojciechowski et al., 1979). The molecular mass for the eggplant enzyme was established as $120 \mathrm{kDa}$. Apparent $K_{m}$ values for sitosterol range from $5.0 \mu \mathrm{M}$ (white mustard seedlings GTase (Wojciechowski et al., 1979)) to $200 \mu \mathrm{M}$ (etiolated maize coleoptyle GTase (Ullmann et al., 1993)), while for UDP-glucose they range from 6.3 $\mu \mathrm{M}$ (potato plant GTase (Zimowski 1992)) to 130 $\mu \mathrm{M}$ (Physarum polycephalum GT-ase (Wojciechowski et al., 1979)). The eggplant enzyme shows the highest affinity for the sugar acceptor among all GTases studied. The $K_{m}$ value for sitosterol, $1.43 \mu \mathrm{M}$, is lower than that for the white mustard seedling enzyme (3-fold).

The influence of lipids on the activity of membrane-bound glycosyltransferases is a well-known phenomenon (Curruthers \& Melchior, 1986). Stimulatory or inhibitory effects are not surprising since lipids make a natural environment for these membrane-bound enzymes. A lipid dependence has also been shown for UDP-glucose : sterol GTases isolated from several plants. It was observed that treatment of membraneous preparations of UDP-glucose : sterol GTase from etiolated pea seedlings with phospholipases caused a partial loss of the enzymatic activity (Fang \& Baisted, 1976), which could be re- stored by some phospholipids (especially phosphatidylethanolamine). Phospholipid influence on UDPglucose : sterol GTase activity was also observed for partly and completely delipidated enzyme preparation from etiolated maize coleoptiles (BouvierNavé et al., 1984; Ullmann et al., 1984). Negatively charged phospholipids, i.e. phosphatidic acid, phosphatidylglycerol, phosphatidylinositol were much more effective than zwitterionic phospholipids, e.g. phosphatydylcholine and phosphatidyletanolamine (Ullmann et al., 1987). We observed a similar influence of negatively charged and zwitterionic phospholipids on eggplant GTase. In addition, eggplant GTase activity was modified (inactivated) in the presence of acylglycerols. It is interesting that some of them, i.e. 1,2-diacylglycerols, also play a role in membraneous secondary messenger signalling pathways. The results of the above experiments strongly suggest the possibility of in vivo regulation of plant UDP-glucose : sterol GTase activity by natural lipid microenvironment.

The substrate specificity of eggplant GTase towards sugar acceptors and sugar donors is similar to those observed for sterol glucosyltransferases from Calendula officinalis, Sinapis alba, Asparagus plumosus, Physarum polycephalum or Solanum tuberosum (Wojciechowski \& Nguyen Van Uon, 1975; Wojciechowski et al., 1979; Pączkowski et al., 1990; Zimowski, 1992). The presence of a double bond at $\mathrm{C}-5$ or the trans coupling of rings $\mathrm{A}$ and $B$, which are prerequisites for the planeness of the sterol ring system, as well as $\beta$ configuration of the 3-OH group are necessary for the activity of all of the investigated enzyme. On the other hand, we have shown that incorporation of the $\mathrm{SH}-$ group at position C-3 $\beta$ makes glucosylation of such a thiol steryl derivative impossible. All sterol GTases seem to exhibit a rather broad specificity pattern if a planar ring system and the $\beta$ configuration of the $3-\mathrm{OH}$ group is fulfilled. Glucosylation rate can be influenced by several structural factors in the sterol molecule such as the presence and/or the position of a double bond, substitution at the side chain or at the ring system and modification of side chain (Yoshikawa \& Furuya, 1979; Zimowski, 1992). It is very interesting that the cytosolic eggplant UDP-glucose: solasodine GTase which also catalyzes the glucosylation of steroids, i.e. steroidal alkaloids and sapogenins, practically does not galactosylate sterols (Pączkowski \& Wojciechowski, 1994).

The substrate specificity of plant UDP-glucose : sterol GTase towards the sugar moiety donor was studied by several researchers (Forsee et al., 1974; Wojciechowski \& Nguyen Van Uon, 1975; Hopp et al., 1978; Wojciechowski et al., 1979; Warnecke \& Heinz, 1994; Warnecke et al., 1997). In 
all cases UDP-glucose was definitely the best sugar donor. Other nucleosidediphosphate glycosides were much poorer substrates for the enzyme. For the eggplant enzyme UDP-glucose is also the best glucose donor, but relatively high glycosylation rates were observed with UDP-galactose, TDPglucose and CDP-glucose. However, the fact that when UDP-galactose was used as a substrate the cholesterol glycosylation product could not be separated from synthetic cholesteryl glucoside by TLC in several solvent systems or by HPLC (unpublished) suggests that UDP-galactose utilization is preceeded by epimerization to UDP-glucose by 4 '-epimerase present in the enzyme preparation. Besides, we could not confirm in vivo sterol galactosylation or the occurrence of sterol galactosides (Zimowski \& Wojciechowski, 1996) in eggplant.

Several genes of plant GT-ases catalyzing nucleotide-sugar-dependent glycosylation of various secondary metabolites have been cloned recently. All these genes contain a consensus sequence, so called PSPG-box (putative secondary product glycosyltransferase-box) (Vogt \& Jones, 2000). This PSPG-box is considered to represent the nucleotide-sugar binding site and contains highly conserved histidine and glutamic acid residues which are proposed to be involved in the transfer of the sugar moiety from the nucleotidesugar donor to the sugar acceptor. Our results confirm that lysine, tryptophan, cysteine, histidine and dicarboxylic amino-acid residues are essential for steryl glucoside formation catalyzed by UDPglucose : sterol GTase from S. melongena. They also indicate that glutamic (or aspartic) acid residue(s) is necessary for binding of sugar donor, i.e. UDPglucose, in the active site of the enzyme and that two other amino acids, i.e. histidine and cysteine are important for the binding of nucleotide-sugar and of the steroidal aglycone. Similar data concerning essential amino-acid residues in the active site of cytosolic UDP-glucose : solasodine GTase from S. melongena have already been obtained. In that case lysine, tryptophan, tyrosine, cysteine, histidine and dicarboxylic amino acid residues of the enzyme are indispensable for glucosylation of steroidal alkaloids. However, only the presence of a histidine residue(s) (for binding sugar acceptor) and a dicarboxylic amino-acid residue(s) (for binding both sugar donor and its acceptor) were required in active site of the enzyme (Nawłoka et al., 2003).

The classical biochemical investigations presented in these paper supply data necessary to study regulation of the activity of plant membraneous enzymes as well as their role in changing the properties of plant membranes stress.

\section{Acknowledgements}

This work was supported in part by research grants BW 1455/11/99 and 1561/54/02 from the Faculty of Biology, Warsaw University, awarded to A.P.

\section{REFERENCES}

Baisted DJ (1978) Steryl glucoside and acyl steryl glucoside biosynthesis in maturing pea seeds. Phytochemistry 17: 435-438.

Bouvier-Navé P, Ullmann P, Rimmele D, Benveniste P (1984) Phospholipid-dependence of plant UDP-glucosesterol- $\beta$-D-glucosyltransferase. I. Detergent-mediated delipidation by selective solubilization. Plant Sci Lett 36: 19-27.

Cantatore JL, Murphy SM, Lynch DV (2000) Compartmentation and topology of glucosylceramide synthesis. Biochem Soc Transact 28: 748-750.

Casabuono AC, Pomilio AB (1997) Linear, steroidal, and triterpene esters, and steryl glycosides from Festuca argentina. Lipids 32: 205-210.

Curruthers A, Melchior DL (1986) How bilayer lipids affect membrane protein activity. Trends Biochem Sci 11: 331-335.

Duperon R, Duperon P (1985) Occurrence of steryl glucosides and steryl galactosides and associated enzyme systems in the leaves of tomato (Solanum lycopersicum L., Solanaceae). C R Acad Sci Paris Serie III 301: 759762.

Duperon R, Duperon P (1989) La glycosylation des stérols chez diverses espèces de mono et dicotylédones: coexistence fréquente de glucosides et de galactosides stéroliques dans les tissus foliaires. $C R$ Acad Sci Paris Serie III 308: 31-34.

Duperon R, Thiersault M, Duperon P (1984) High level of glycosylated sterols in species of Solanum and sterol changes during the development of the tomato. Phytochemistry 23: 743-746.

Esders TW, Light RJ (1972) Occurrence of a uridine diphosphate glucose: sterol glucosyltransferase in Candida bogoriensis. J Biol Chem 247: 248-259.

Fang T-Y, Baisted DJ (1976) UDPG : sterol glucosyltransferase in etiolated pea seedlings. Phytochemistry 15: 273-278.

Forsee WT, Laine RA, Elbein AD (1974) Solubilization of a particulate UDP-glucose : sterol $\beta$-D-glucosyltransferase in developing cotton fibres and seeds and characterization of steryl 6-acyl-D-glucosides. Arch Biochem Biophys 161: 248-259.

Fujino Y, Ohnishi M (1979) Isolation and structure of diglycosylsterols and triglycosylsterols in rice bran. Biochim Biophys Acta 574: 94-102.

Hopp HE, Romero PA, Daleo GR, Lezica RP (1978) Steryl glucoside biosynthesis in the alga Prototheca zopfii. Phytochemistry 17: 1049-1052.

Hou CT, Umemura M, Nakamura M, Funahashi S (1967) Enzymatic synthesis of steryl glucoside by a particulare preparation from immature soybean seeds. J Biochemistry (Tokyo) 62: 389-391.

Jahan N, Ahmed W, Malik A (1995) A new steroidal glycosides from Mimusops elengi. J Nat Prod 58: 1244-1247.

Janiszowska W, Wiłkomirski B, Kasprzyk Z (1980) Synthesis of oleanolic acid 3-O-monoglucoside. Pol J Chem 54: 2147-2152. 
Jares EA, Tettamanzi MC, Pomilio AB (1990) Sitosterol 3O- $\beta$-D-glucuronopyranoside from Senecio bonariensis. Phytochemistry 29: 340-341.

Kojima M, Ohnishi M, Ito S, Fujino Y (1989) Characterization of acylmono-, mono-, di-, tri- and tetraglycosylsterol and saponin in adzuki bean (Vigna angularis) seeds. Lipids 24: 849-856.

Murakami-Murofushi K, Nishikawa K, Hirakawa E, Murofushi H (1997) Heat stress induces a glycosylation of membrane sterol in myxoamoebae of a true slime mold, Physarum polycephalum. J Biol Chem 272: 486-489.

Nawłoka P, Kalinowska M, Pączkowski C, Wojciechowski ZA (2003) Evidence for essential histidine and dicarboxylic amino-acid residues in the active site of UDPglucose: solasodine glucosyltransferase from eggplant leaves. Acta Biochim Polon 50: 567-572.

Pączkowski C, Wojciechowski ZA (1994) Glucosylation and galactosylation of diosgenin and solasodine by soluble glycosyltransferase(s) from Solanum melongena leaves. Phytochemistry 35: 1429-1434.

Pączkowski C, Zimowski J, Krawczyk D, Wojciechowski ZA (1990) Steroid-specific glucosyltransferases in Asparagus plumosus shoots. Phytochemistry 29: 63-70.

Peng L, Kawagoe Y, Hogan P, Delmer D (2002) Sitosterol$\beta$-glucoside as primer for cellulose synthesis in plants. Science 295: 147-150.

Peters WS (2002) The lipid origin of cellulose. Trends Cell Biol 12: 159.

Potocka A, Zimowski J (2008) Metabolism of conjugated sterols in eggplant. Part 2. Phospholipid : steryl glucoside acyltransferase. Acta Biochim Polon 55: 135-140.

Tannes T, Grav HJ, Bukholm G (2000) Lipid profiles of Helicobacter pylori colony variants. APMIS 108: 349-356.

Ullmann P, Rimmele D, Benveniste P, Bouvier-Navé P (1984) Phospholipid-dependence of plant UDP-glucose-sterol- $\beta$-D-glucosyltransferase. II. Acetone-mediated delipidation and kinetic studies. Plant Sci Lett 36: 29-36.

Ullmann P, Bouvier-Navé P, Benveniste P (1987) Regulation by phospholipids and kinetic studies of plant membrane-bound UDP-glucose sterol $\beta$-D-glucosyl transferase. Plant Physiol 85: 51-55.

Ullmann P, Ury A, Rimmele D, Benveniste P, BouvierNavé P (1993) UDP-glucose sterol $\beta$-D-glucosyltransferase, a plasma membrane-bound enzyme of plants: enzymatic properties and lipid dependence. Biochimie 75: 713-723.

Vogt T, Jones P (2000) Glycosyltransferases in plant natural product synthesis: characterization of a supergene family. Trends Plant Sci 5: 380-386.

Warnecke DC, Baltrusch M, Buck F, Wolter FP, Heinz E (1997) UDP-glucose : sterol $\beta$-D-glucosyltransferase: cloning and functional expression in Escherichia coli. Plant Mol Biol 35: 597-603.

Warnecke D, Erdmann R, Fahl A, Hube B, Müller F, Zank T, Zähringer U, Heinz E (1999) Cloning and functional expression of ugt genes encoding sterol glucosyltransferases from Saccharomyces cerevisiae, Candida albicans, Pichia pastoris, and Dictyostelium discoideum. J Biol Chem 274: 13048-13059.

Warnecke DC, Heinz E (1994) Purification of membranebound UDP-glucose : sterol $\beta$-D-glucosyltransferase based on its solubility in diethyl ether. Plant Physiol 105: 1067-1073.

Wojciechowski ZA (1991) Biochemistry of phytosterols conjugates. In Physiology and Biochemistry of Sterols. Patterson GW, Nes WD, eds, pp 361-393. AOCS, Champaign, Il.

Wojciechowski ZA, Nguyen Van Uon (1975) Intracellular localization and some properties of UDPG : sterol glucosyltransferase from Calendula officinalis. Acta Biochim Polon 22: 25-38.

Wojciechowski ZA, Zimowski J, Zimowski JG, Łyżnik A (1979) Specificity of sterol-glucosylating enzymes from Sinapis alba and Physarum polycephalum. Biochim Biophys Acta 570: 363-370.

Yoshikawa T, Furuya T (1979) Purification and properties of sterol: UDPG glucosyltransferase in cell culture of Digitalis purpurea. Phytochemistry 18: 239-241.

Zimowski J (1991) The occurrence of a glucosyltransferase specific for solanidine in potato plants. Phytochemistry 30: 1187-1193.

Zimowski J (1992) Specificity and some other properties of cytosolic and membraneous UDPGLC : $3 \beta$-hydroxysteroid glucosyltransferases from Solanum tuberosum leaves. Phytochemistry 31: 2977-2981.

Zimowski J, Wojciechowski ZA (1996) TLC and GLC Analysis of individual molecular species in natural mixtures of steryl glycosides isolated from eggplant. Herba Polon 4: 296-272. 